\title{
Factors Governing the Different Functions of Zn2+-Sites with Identical Ligands in Proteins
}

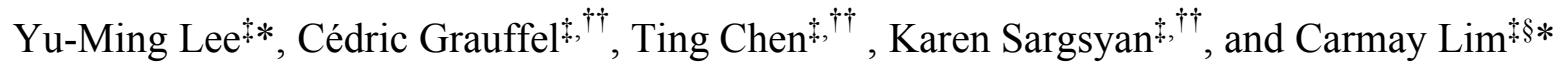

Hnstitute of Biomedical Sciences, Academia Sinica, Taipei 115, Taiwan

$\S$ Department of Chemistry, National Tsing Hua University, Hsinchu 300 Taiwan

\footnotetext{
*E-mail: leon@ibms.sinica.edu.tw

*E-mail: carmay@gate.sinica.edu.tw
} 
Table S1. Non-redundant $\mathrm{Zn}-\mathrm{CC}(\mathrm{C} / \mathrm{H}) \mathrm{x}\left(\mathrm{x}=\mathrm{D}, \mathrm{E}\right.$ or $\left.\mathrm{H}_{2} \mathrm{O}\right)$ sites in the PDB (2019, February). ${ }^{\mathrm{a}}$

\begin{tabular}{|c|c|c|c|c|}
\hline $\begin{array}{c}\text { Cluster } \\
\#\end{array}$ & PDB IDs & $\begin{array}{c}\text { Representative } \\
\text { Zn-ligands }\end{array}$ & $\begin{array}{c}\text { \# of } \\
\text { Zn-sites }\end{array}$ & Protein description \\
\hline & Archaea & & & \\
\hline 1 & $\begin{array}{l}\text { 1g5c_a, 3ten_a, 3ten_b, } \\
\text { 3ten_c, 3ten_d, 3ten_e, } \\
\text { 3ten_f, 3ten_g, 3ten_h, } \\
\text { 1g5c_b, 1g5c_c, 1g5c_d, } \\
\text { 1g5c_e, 1g5c_f }\end{array}$ & $\mathrm{CCHw}$ & 14 & $\beta$-carbonic anhydrase \\
\hline 2 & $\begin{array}{l}\text { 1h2b_b, 1jvb_a*, 1nto_a*, } \\
\text { 1nto_b*, 1nto_c*, } \\
\text { 1nto_d*, 1nto_e*, } \\
\text { 1nto_h*, 1nvg_a*, 1r37_a, } \\
\text { 1r37_b, 2eer_a, 2eer_b, } \\
\text { 3i4c_a*, 3i4c_b*, 3i4c_c*, } \\
\text { 3i4c_d*, 3i4c_e*, 3i4c_h*, } \\
\text { 2h6e_a }\end{array}$ & CCCD & 34 & $\begin{array}{l}\text { Alcohol } \\
\text { dehydrogenase }\end{array}$ \\
\hline 3 & 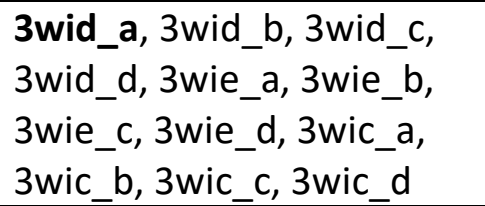 & CCCD & 12 & $\begin{array}{l}\text { Glucose 1- } \\
\text { dehydrogenase }\end{array}$ \\
\hline 4 & 4ay7_b & $\mathrm{CCHE}$ & 1 & Methyltransferase \\
\hline 5 & 1zt2_a, 1zt2_c & CCCD & 2 & $\begin{array}{l}\text { DNA primase small } \\
\text { subunit }\end{array}$ \\
\hline 6 & 2gvi_a & CCCD & 1 & $\begin{array}{l}\text { Conserved } \\
\text { hypothetical protein }\end{array}$ \\
\hline \multirow[t]{2}{*}{7} & 2oso_a, 2osd_a & $\mathrm{CCHE}$ & 2 & $\begin{array}{l}\text { Hypothetical protein } \\
\text { mj1460 }\end{array}$ \\
\hline & Bacteria & & & \\
\hline 1 & 1ctt_a, 1aln_a & $\mathrm{CCHw}$ & 2 & Cytidine deaminase \\
\hline 2 & $\begin{array}{l}\text { 1fbx_a, 1fbx_b, 1fbx_c, } \\
\text { 1fbx_d, 1fbx_e, 1fbx_f, } \\
\text { 1fbx_h, 1fbx_i, 1fbx_j, } \\
\text { 1fbx_l, 1fbx_n, 1fbx_o }\end{array}$ & $\mathrm{CCHw}$ & 12 & GTP cyclohydrolase i \\
\hline 3 & $\begin{array}{l}\text { 116s_a, 1w5o_a, 1b4e_a, } \\
1 i 8 j \_a, 1 i 8 j \_b, 116 s \_b, \\
116 y \_a, 116 y \_b\end{array}$ & $\mathrm{CCCW}$ & 8 & $\begin{array}{l}\delta \text {-aminolevulinic acid } \\
\text { dehydratase }\end{array}$ \\
\hline 4 & $\begin{array}{l}\text { 1li5_a, 3sp1_a, 3sp1_b, } \\
\text { 3tqo_a, 1li5_b }\end{array}$ & $\mathrm{CCHE}$ & 5 & $\begin{array}{l}\text { Cysteinyl-tRNA } \\
\text { synthetase }\end{array}$ \\
\hline 5 & 1uuf_a & $\mathrm{CCHw}$ & 1 & $\begin{array}{l}\text { Alcohol } \\
\text { dehydrogenase-like } \\
\text { protein yahk }\end{array}$ \\
\hline 6 & 1wkq_a, 1wkq_b & $\mathrm{CCHw}$ & 2 & Guanine deaminase \\
\hline 7 & $\begin{array}{l}\text { 1wwr_a, 2hvv_a, 2hvv_b, } \\
\text { 5c2o_a, 5c2o_b, 1wwr_b, } \\
\text { 1wwr_c, 1wwr_d }\end{array}$ & $\mathrm{CCHw}$ & 8 & $\begin{array}{l}\text { tRNA-specific } \\
\text { adenosine }\end{array}$ \\
\hline
\end{tabular}




\begin{tabular}{|c|c|c|c|c|}
\hline 8 & 1ym3_a & CCHD & 1 & $\beta$-carbonic anhydrase \\
\hline 9 & $\begin{array}{l}\text { 2b3j_a, 2b3j_b, 2b3j_c, } \\
2 b 3 j \_d\end{array}$ & $\mathrm{CCHw}$ & 4 & $\begin{array}{l}\text { tRNA-specific } \\
\text { adenosine } \\
\text { deaminase }\end{array}$ \\
\hline 10 & 2bz1_a & $\mathrm{CCCW}$ & 1 & GTP cyclohydrolase ii \\
\hline 11 & 2fgy_a, 2fgy_b & $\mathrm{CCHw}$ & 2 & $\beta$-carbonic anhydrase \\
\hline 12 & 2g0d_a, 2g02_a & $\mathrm{CCHw}$ & 2 & $\begin{array}{l}\text { Nisin biosynthesis } \\
\text { protein NisC }\end{array}$ \\
\hline 13 & $\begin{array}{l}\text { 2g84_a, 2g84_b, 4hrq_a, } \\
\text { 4hrq_b, 4lc5_a, 4lc5_b, } \\
\text { 4lcn_a, 4lcn_b, 4lco_a, } \\
\text { 4lco_b, 4lcp_a, 4lcp_b, } \\
\text { 4ld2_a, 4ld2_b }\end{array}$ & $\mathrm{CCHw}$ & 14 & $\begin{array}{l}\text { Cytidine and } \\
\text { deoxycytidylate } \\
\text { deaminase }\end{array}$ \\
\hline 14 & $\begin{array}{l}\text { 2hxv_a, 3zpc_b, 2b3z_a, } \\
2 b 3 z \_b, 3 e x 8 \_b, 30 c q \_a, \\
\text { 1z3a_a, 1z3a_b }\end{array}$ & $\mathrm{CCHw}$ & 8 & $\begin{array}{l}\text { Riboflavin biosynthesis } \\
\text { protein RibD }\end{array}$ \\
\hline 15 & $\begin{array}{l}\text { 3bq6_a, 3t0c_a, 3bq6_b, } \\
\text { 1xpg_a, 1xpg_b }\end{array}$ & $\mathrm{CCHE}$ & 5 & $\begin{array}{l}\text { homocysteine } \\
\text { methyltransferase }\end{array}$ \\
\hline 16 &  & $\mathrm{CCCW}$ & 18 & Cytidine deaminase \\
\hline 17 & 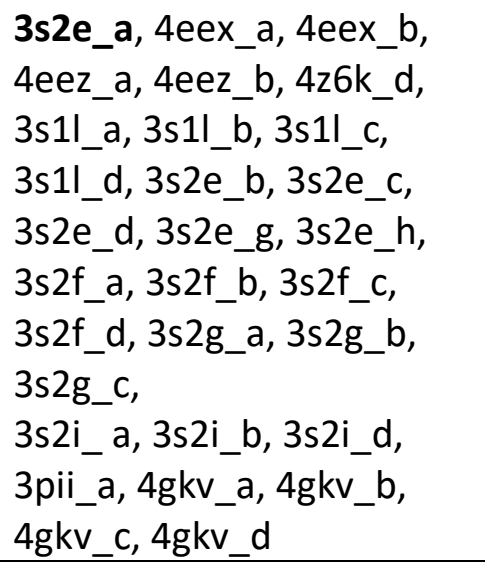 & $\mathrm{CCHE}$ & 30 & $\begin{array}{l}\text { Alcohol } \\
\text { dehydrogenase }\end{array}$ \\
\hline 18 & $\begin{array}{l}\text { 3vrk_a, 1ylk_a, 1ylk_b, } \\
\text { 1ylk_d, 3las_a, 3las_b }\end{array}$ & $\mathrm{CCHw}$ & 6 & $\begin{array}{l}\text { Carbonyl sulfide } \\
\text { hydrolase }\end{array}$ \\
\hline 19 & 3wrg_a, 3wre_a & CCCE & 2 & $\begin{array}{l}\text { Non-reducing end } \beta \mathrm{I}- \\
\text { arabinofuranosidase }\end{array}$ \\
\hline 20 & $\begin{array}{l}\text { 5cxk_a, 2a8c_a, 2a8c_b, } \\
\text { 2a8c_c, 2a8c_d, 2a8c_e, } \\
\text { 2a8c_f, 2a8d_a, 2a8d_b, } \\
\text { 2a8d_c, 2a8d_d, 2a8d_e, } \\
\text { 2a8d_f, 3e24_a, 3e28_b, } \\
\text { 3e28_c, 3e28_d, 3e2a_a, } \\
\text { 3e2a_b, 3e2a_c, 3e2a_d, }\end{array}$ & $\mathrm{CCHD}$ & 74 & $\beta$-carbonic anhydrase \\
\hline
\end{tabular}




\begin{tabular}{|c|c|c|c|c|}
\hline & $\begin{array}{l}\text { 3e2a_e, 3e2a_f, 3e2w_a, } \\
\text { 3e2w_b, 3e2w_c, 3e2w_d, } \\
\text { 3e2x_a, 3e2x_b, 3e31_a, } \\
\text { 3e31_b, 3e3f_b, 3e3g_a, } \\
\text { 3e3g_b, 3e3g_c, 3e3g_d, } \\
\text { 3e3g_e, 3e3g_f, 3e3i_a, } \\
\text { 3e3i_b, 3e3i_c, 3e3i_d, } \\
\text { 3e3i_e, 3e3i_f, 3e3i_g, } \\
\text { 3e3i_h, 3e3i_i, 3e3i_j, } \\
\text { 3e3i_k, 3e3i_l, 4waj_a, } \\
\text { 4waj_b, 4wak_a, 4wak_b, } \\
\text { 4wam_b, 5cxk_b, 5cxk_c, } \\
\text { 5cxk_d, 5cxk_e, 5cxk_f, } \\
\text { 5cxk_g, 5cxk_h, 1i6o_a, } \\
\text { 1i6o_b, 1i6p_a, 1t75_a, } \\
\text { 1t75_b, 1t75_d, 1t75_e, } \\
\text { 2esf_a, 2esf_b, 4znz_a, } \\
\text { 4rxy_a, 5bq1_a }\end{array}$ & & & \\
\hline 21 & 5k1s_d & $\mathrm{CCHE}$ & 1 & $\begin{array}{l}\text { Zinc-binding } \\
\text { dehydrogenase }\end{array}$ \\
\hline 22 & $\begin{array}{l}\text { 5swc_a, 5swc_b, 5swc_c, } \\
5 s w c \_d, 5 s w c \_e, 5 s w c \_f\end{array}$ & $\mathrm{CCHW}$ & 6 & $\beta$-carbonic anhydrase \\
\hline 23 & $4060 \_b$ & $\mathrm{CCCW}$ & 1 & $\begin{array}{l}\text { Recombination protein } \\
\text { RecR }\end{array}$ \\
\hline 24 & $\begin{array}{l}\text { 5fru_a, 5kbe_b, 5kbg_b, } \\
5 \mathrm{kbh} \text {-a, 5kbh_b, 5kbi_a, } \\
5 f r u \_b, 5 f r v \_a, 5 f r v \_b, \\
5 f r w \_a, 5 f r w \_b, 5 f r x \_a, \\
5 f r y \_a, 5 f r y \_b, 5 f r z \_a, \\
5 f r z \_b, 5 f s 0 \_a, 5 f s 0 \_b\end{array}$ & CCCE & 18 & $\begin{array}{l}\text { Positive phenol- } \\
\text { degradative } \\
\text { gene regulator }\end{array}$ \\
\hline 25 & 2hf1_a, 2hf1_b & CCCD & 2 & $\begin{array}{l}\text { Putative } \\
\text { tetraacyldisaccharide- } \\
\text { 1-p 4-kinase }\end{array}$ \\
\hline 26 & $\begin{array}{l}\text { 2qq4_a, 2qq4_b, 2qq4_c, } \\
\text { 2qq4_d, 2qq4_e, 2qq4_f }\end{array}$ & CCCD & 6 & $\begin{array}{l}\text { Iron-sulfur } \\
\text { biosynthesis protein }\end{array}$ \\
\hline 27 & 3di4_a, 3di4_b & $\mathrm{CCCW}$ & 2 & $\begin{array}{l}\text { DUF1989 family } \\
\text { protein }\end{array}$ \\
\hline 28 & $\begin{array}{l}\text { 3oru_a, 3siy_a, 3siy_b, } \\
\text { 3siy_c, 3siy_d }\end{array}$ & $\mathrm{CCCW}$ & 5 & $\begin{array}{l}\text { DUF1989 family } \\
\text { protein }\end{array}$ \\
\hline 29 & $\begin{array}{l}\text { 3p1v_a, 4df9_a, 4df9_b, } \\
\text { 4df9_c, 4df9_d, 4df9_e, } \\
\text { 4df9_f, 3p1v_b }\end{array}$ & CCCE & 8 & $\begin{array}{l}\text { Metallo- } \\
\text { endopeptidase }\end{array}$ \\
\hline 30 & 4esn_a, 4esn_b & $\mathrm{CCCW}$ & 2 & $\begin{array}{l}\text { DUF1312 family } \\
\text { protein }\end{array}$ \\
\hline 31 & $\frac{4 q b g \_b}{3 d l 0 \_b}, 4 t y p \_b, 3 d l 0 \_a$, & CCCD & 4 & Adenylate kinase \\
\hline
\end{tabular}




\begin{tabular}{|c|c|c|c|c|}
\hline & Eukaryotes & & & \\
\hline 1 & 1ekj_c & $\mathrm{CCHw}$ & 1 & $\begin{array}{l}\text { Pisum sativum } \beta \\
\text { carbonic anhydrase }\end{array}$ \\
\hline 2 & $\begin{array}{l}\text { 1h7n_a, 1h7p_a, 1h7r_a, } \\
\text { 1w31_a, 1h7o_a, 1eb3_a, } \\
\text { 1gjp_a, 5hnr_a }\end{array}$ & $\mathrm{CCCW}$ & 8 & $\begin{array}{l}\text { 5-aminolaevulinic acid } \\
\text { dehydratase }\end{array}$ \\
\hline 3 & $\begin{array}{l}\text { 1is8_c, 1is8_b, } \\
\text { 1is8_e,1is8_f, 1is8_g, } \\
\text { 1is8_h }\end{array}$ & $\mathrm{CCHw}$ & 6 & Cytidine deaminase \\
\hline 4 & $\begin{array}{l}\text { 1mgo_a, 4dl9_a, 4dl9_b, } \\
\text { 4dla_a, 4dla_b, 4dlb_a, } \\
\text { 3uko_a, 3uko_b, 4g|4_a, } \\
\text { 4gl4_b, 4jji_b, 4l0q_a, } \\
\text { 4l0q_b, 1e3e_a, 1e3e_b, } \\
\text { 1e3l_a, 1e3l_b, 4rqu_b, } \\
\text { 1adf_a, 1adg_a, 1cdo_b, } \\
\text { 1hso_a, 1hso_b, 1hsz_a, } \\
\text { 1ht0_a, 1ht0_b, 1ju9_a, } \\
\text { 1ju9_b, 1mc5_b, 1mgo_b, } \\
\text { 1qlh_a, 1qlj_a, 1ye3_a, } \\
\text { 5adh_a, 8adh_a, 1m6h_a, } \\
\text { 1m6h_b, 1ma0_a, } \\
\text { 1ma0_b, 1mp0_a, 1teh_b, } \\
\text { 2few_a, 2fzw_b }\end{array}$ & $\mathrm{CCHw}$ & 43 & $\begin{array}{l}\text { Alcohol } \\
\text { dehydrogenase E chain }\end{array}$ \\
\hline 5 & $\begin{array}{l}\text { 1r5t_a, 1zab_a, 1zab_b, } \\
\text { 1zab_c, 1zab_d, 2fr6_a, } \\
\text { 2fr6_c, 2fr6_d, 1r5t_b, } \\
\text { 1r5t_c, 1r5t_d, 1mq0_b }\end{array}$ & $\mathrm{CCCW}$ & 12 & Cytidine deaminase \\
\hline 6 & $\begin{array}{l}\text { 1zy7_a, 1zy7_b, 5ed1_d, } \\
\text { 5hp2_d, 5hp3_d }\end{array}$ & $\mathrm{CCHw}$ & 5 & adenosine deaminase \\
\hline 7 & 2w3q_a & $\mathrm{CCHw}$ & 1 & $\begin{array}{l}\beta \text {-carbonic anhydrase } \\
2\end{array}$ \\
\hline 8 & 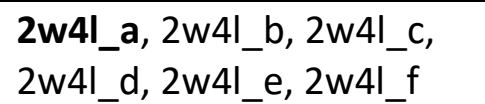 & $\mathrm{CCHw}$ & 6 & $\begin{array}{l}\text { Deoxycytidylate } \\
\text { deaminase }\end{array}$ \\
\hline 9 & $\begin{array}{l}\text { 2z3g_a, 2z3g_b, 2z3g_c, } \\
2 z 3 g \_d, 2 z 3 h \_d\end{array}$ & CCCW & 5 & $\begin{array}{l}\text { Blasticidin S } \\
\text { Deaminase }\end{array}$ \\
\hline 10 & 3boc_a & $\mathrm{CCHw}$ & 1 & $\begin{array}{l}\text { Carbonic anhydrase of } \\
\text { marine diatoms }\end{array}$ \\
\hline 11 & 3dh1_a & $\mathrm{CCHw}$ & 1 & $\begin{array}{l}\text { tRNA-specific } \\
\text { adenosine } \\
\text { deaminase } 2 \\
\end{array}$ \\
\hline 12 & $3 e 6 u \_d$ & $\mathrm{CCHw}$ & 1 & $\begin{array}{l}\text { Eukaryotic lanthionine } \\
\text { synthetase C-like } \\
\text { protein } 1\end{array}$ \\
\hline 13 & $\begin{array}{l}\text { 3eyx_a, 1ddz_a*, 1ddz_b*, } \\
\text { 3uco_a, 3uco_b, 3eyx_b }\end{array}$ & $\mathrm{CCHw}$ & 8 & $\beta$-carbonic anhydrase \\
\hline
\end{tabular}




\begin{tabular}{|c|c|c|c|c|}
\hline 14 & $\begin{array}{l}\text { 3v4k_a, 5jj4_a, 5jj4_b, } \\
\text { 5jj4_c, 3ir2_a, 3ir2_b, } \\
\text { 3v4k_b, 5hx5_a, 4rov_a, } \\
\text { 4rov_b, 4row_a, 5k81_a, } \\
\text { 5k81_b, 5k81_c, 5k81_d, } \\
\text { 5k81_e, 5k81_f, 5k83_a, } \\
\text { 5k83_b, 5k83_c, 5k83_d, } \\
\text { 5k83_e, 5k83_f, 2nyt_a, } \\
\text { 2nyt_c, 3vow_b, 3wus_a, } \\
\text { 3wus_b, 3e1u_a, 3iqs_a, } \\
\text { 5cqd_a, 5cqd_c, 5cqi_a, } \\
\text { 5cqk_a }\end{array}$ & $\mathrm{CCHw}$ & 34 & $\begin{array}{l}\text { DNA dC } \rightarrow \text { dU-editing } \\
\text { enzyme APOBEC-3G }\end{array}$ \\
\hline 15 & $\begin{array}{l}\text { 416o_a, 3ppg_a, 4l61_a, } \\
4164 \_a, 4 z t x \_a, 1 u 1 h \_a, \\
1 u 1 j \_a\end{array}$ & $\mathrm{CCHw}$ & 7 & $\begin{array}{l}\text { homocysteine } \\
\text { methyltransferase } \\
\text { (Met6p) }\end{array}$ \\
\hline 16 & $4 w 6 z \_b, 4 w 6 z \_d$ & $\mathrm{CCHE}$ & 2 & $\begin{array}{l}\text { Yeast alcohol } \\
\text { dehydrogenase }\end{array}$ \\
\hline 17 & $\begin{array}{l}\text { 5b5z_a, 5b5y_a, 5b5y_b, } \\
5 b 5 z \text { b, 5b60_a }\end{array}$ & $\mathrm{CCHw}$ & 5 & $\beta$-carbonic anhydrases \\
\hline 18 & $\begin{array}{l}\text { 5fi3_a, 5h81_a, 5h81_b, } \\
\text { 5h82_a, 5h82_b, 1yqd_a, } \\
\text { 1yqd_b, 1yqx_b, 5h83_a, } \\
\text { 5h83_b, 5fi3_b, 5fi5_a, } \\
5 f i 5 \_b\end{array}$ & $\mathrm{CCHE}$ & 13 & $\begin{array}{l}\text { Tetrahydroalstonine } \\
\text { synthase }\end{array}$ \\
\hline 19 & $5 k 5 w \_a, 5 k 5 w \_b$ & $\mathrm{CCHw}$ & 2 & $\beta$-carbonic anhydrases \\
\hline 20 & 1du3_k & $\mathrm{CCCW}$ & 1 & TRAIL-DR5 complex \\
\hline 21 & 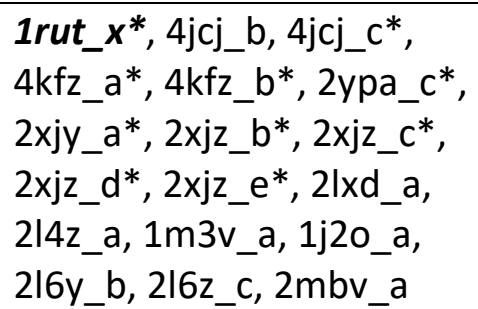 & CCCD & 28 & $\begin{array}{l}\text { LIM domain-binding } \\
\text { protein } 1\end{array}$ \\
\hline 22 & 2dar_a, 2dlo_a, 2dj7_a & $\mathrm{CCHE}$ & 3 & $\begin{array}{l}\text { Pdz and lim domain } \\
\text { protein } 5\end{array}$ \\
\hline 23 & $\begin{array}{l}\text { 2rgt_a*, 2jtn_a*,2rgt_b*, } \\
\text { 3mmk_a*, 3mmk_b* }\end{array}$ & CCCD & 10 & $\begin{array}{l}\text { Lim domain-binding } \\
\text { protein }\end{array}$ \\
\hline 24 & $\begin{array}{l}\text { 3dpl_d, 1u6g_b, 3rtr_b, } \\
\text { 3rtr_d, 3rtr_f, 3rtr_h, } \\
\text { 2lgv_a, 1ldj_b }\end{array}$ & $\mathrm{CCHD}$ & 8 & $\begin{array}{l}\text { E3 ubiquitin-protein } \\
\text { ligase RBX1 }\end{array}$ \\
\hline 25 & 3hcu_a & $\mathrm{CCHD}$ & 1 & $\begin{array}{l}\text { Tnf receptor- } \\
\text { associated factor } 6 \\
\text { (TRAF6) }\end{array}$ \\
\hline 26 & 3vhs_a & $\mathrm{CCHw}$ & 1 & Atpase wrnip1 \\
\hline 27 & $\begin{array}{l}\text { 4his_b, 3f6q_b, 3ixe_b, } \\
\text { 4hi9_b }\end{array}$ & CCCD & 4 & $\begin{array}{l}\text { Integrin-linked protein } \\
\text { kinase }\end{array}$ \\
\hline
\end{tabular}




\begin{tabular}{|c|c|c|c|c|}
\hline 28 & $\begin{array}{l}\text { 4xba_a, 3szq_a, 3sp4_a, } \\
\text { 3sp4_b, 3spd_a, 3spd_b, } \\
\text { 3spd_c, 3spd_d, 3spl_a, } \\
\text { 3spl_b, 3spl_c, 3spl_d, } \\
\text { 4ykl_b }\end{array}$ & $\mathrm{CCHE}$ & 13 & Aprataxin-like protein \\
\hline 29 & $4 y b g \_a$ & $\mathrm{CCHE}$ & 1 & $\begin{array}{l}\text { Drosophila } \\
\text { melanogaster } \\
\text { maelstrom } \\
\end{array}$ \\
\hline 30 & 5afo_c, 5afo_b, 5afo_d & $\mathrm{CCHE}$ & 3 & $\begin{array}{l}\text { Mael domain from } \\
\text { bombyx } \\
\text { mori maelstrom }\end{array}$ \\
\hline & Virus & & & \\
\hline 1 & 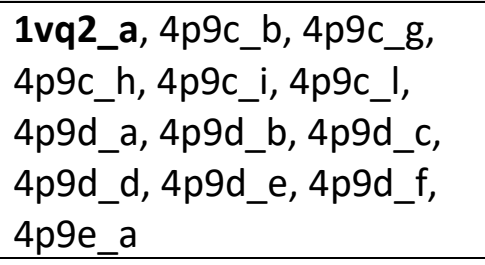 & $\mathrm{CCHE}$ & 13 & $\begin{array}{l}\text { Deoxycytidylate } \\
\text { deaminase }\end{array}$ \\
\hline 2 & $\begin{array}{l}\text { 2g9t_e, 2g9t_a, 2g9t_b, } \\
\text { 2g9t_c, 2g9t_d, 2g9t_f, } \\
\text { 2g9t_g, 2g9t_h, 2g9t_i, } \\
\text { 2g9t_, 2g9t_k, 2g9t_l, } \\
\text { 2g9t_m, 2g9t_n, 2g9t_o, } \\
\text { 2g9t_p, 2g9t_q, 2g9t_r, } \\
\text { 2g9t_s, 2g9t_t, 2g9t_u, } \\
\text { 2g9t_w, 2ga6_e, 2ga6_g, } \\
\text { 2ga6_q, 2ga6_s, 2ga6_t, } \\
\text { 2ga6_v, 2ga6_w, 2xyr_b }\end{array}$ & $\mathrm{CCCW}$ & 30 & Sars coronavirus nsp10 \\
\hline 3 & 2wbt_a, 2wbt_b & $\mathrm{CCHE}$ & 2 & $\begin{array}{l}\text { A double } \mathrm{C}_{2} \mathrm{H}_{2} \text { zinc } \\
\text { finger protein }\end{array}$ \\
\hline 4 & 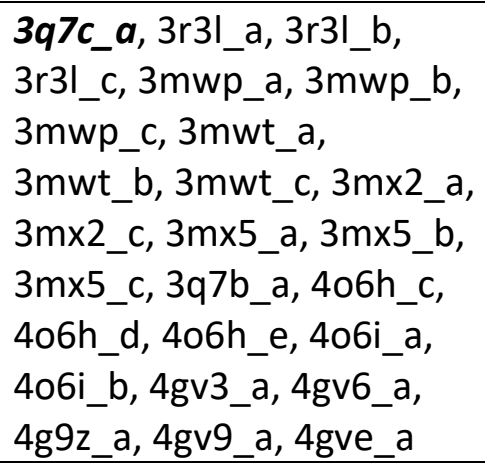 & $\mathrm{CCHE}$ & 26 & $\begin{array}{l}\text { Nucleoprotein } \\
\text { structure of } \\
\text { Lassa fever virus }\end{array}$ \\
\hline 5 & 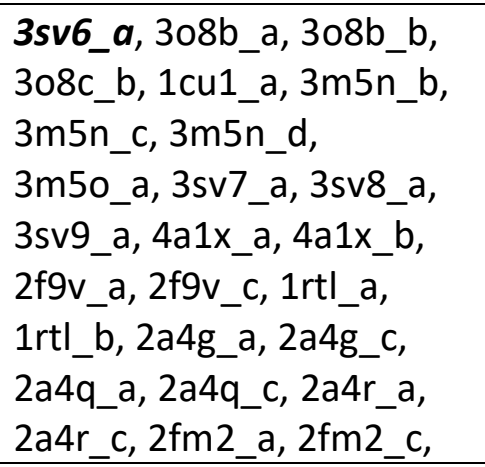 & $\mathrm{CCCW}$ & 74 & $\begin{array}{l}\text { HCV NS3 protease } \\
\text { domain/ } \\
\text { NS4a peptide complex }\end{array}$ \\
\hline
\end{tabular}




\begin{tabular}{|c|c|c|c|c|}
\hline & 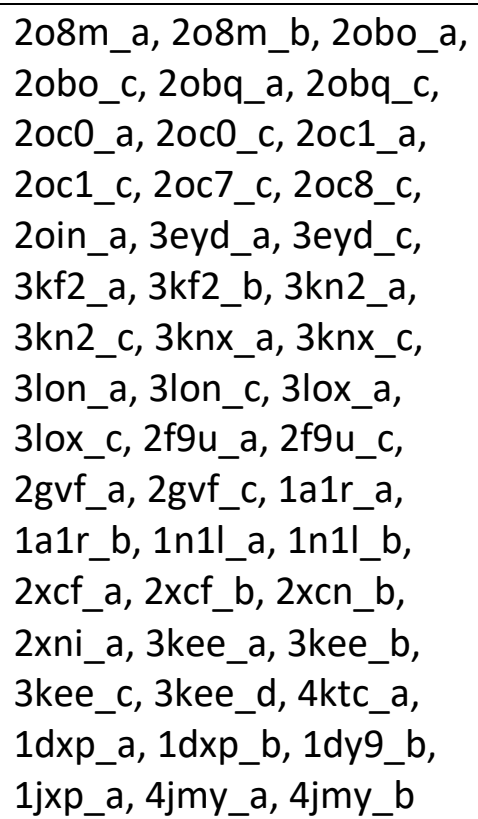 & & & \\
\hline 6 & $\begin{array}{l}\text { 4hhj_a, 4k6m_b*, 5tfr_a, } \\
\text { 5tfr_b, 5ccv_a, 5u0b_a, } \\
\text { 5u0b_b, 5tmh_a, 5tmh_b, } \\
\text { 4v0q_a, 4v0r_a, 5dto_a, } \\
\text { 5jjr_a, 2hfz_a, 4hdh_a, } \\
\text { 4hdh_b, 5u0c_a, 5u0c_b, } \\
\text { 5u0c_d, 5u0c_e, 5u0c_f, } \\
\text { 5u0c_g, 5u0c_h, 4c11_a, } \\
\text { 4c11_b, 3vws_a, 5f3t_a, } \\
\text { 5f3z_a, 5f41_a, 5hmw_a, } \\
\text { 5hmx_a, 5hmy_a, 5hmz_a, } \\
\text { 5hn0_a, 5i3p_a, 5i3q_a, } \\
\text { 5k5m_a, 4mtp_a, 4mtp_b, } \\
4 m t p \_c, 5 w z 3 \text { a*, } \\
\text { 5u04_a* }\end{array}$ & $\mathrm{CCHE}$ & 45 & $\begin{array}{l}\text { RNA-directed RNA } \\
\text { polymerase (NS5) }\end{array}$ \\
\hline
\end{tabular}

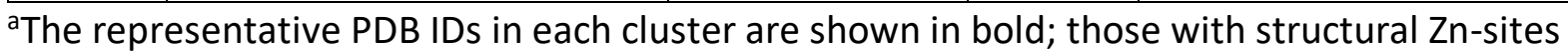
are in italics, and those with $\mathrm{Zn}$-sites of unknown function are underlined. PDB entries with two $\mathrm{Zn}$ sites are labeled " *". 
Table S2. Second-shell residues that form hydrogen bonds with the bound water molecule in $\mathrm{Zn}-\mathrm{CC}(\mathrm{C} / \mathrm{H}) \mathrm{w}$ sites in archaea, bacteria, eukaryotes and viruses.

\begin{tabular}{|c|c|c|c|}
\hline PDB & Zn ligands & Structural/Catalytic & $2^{\text {nd }}$ shell of $\mathrm{Zn}$-bound water \\
\hline $\begin{array}{c}\text { Archaea } \\
\text { 1g5c_a }\end{array}$ & $\mathrm{CCHw}$ & Catalytic & D34 \\
\hline \multicolumn{4}{|l|}{ Bacteria } \\
\hline 2g84_a & $\mathrm{CCHw}$ & Catalytic & E79 \\
\hline 1fbx_a & $\mathrm{CCHw}$ & Catalytic & E111 \\
\hline $2 h x v \_a$ & $\mathrm{CCHw}$ & Catalytic & E51 \\
\hline 1wwr_a & $\mathrm{CCHw}$ & Catalytic & E54 \\
\hline 3vrk_a & $\mathrm{CCHw}$ & Catalytic & D46 \\
\hline 5swc_a & $\mathrm{CCHw}$ & Catalytic & D41 \\
\hline 2b3j_a & $\mathrm{CCHw}$ & Catalytic & E55 \\
\hline 1wkq_a & $\mathrm{CCHw}$ & Catalytic & E55 \\
\hline 2fgy_a & $\mathrm{CCHw}$ & Catalytic & D175 \\
\hline 1ctt_a & $\mathrm{CCHw}$ & Catalytic & E104 \\
\hline 3dmo_a & $\mathrm{CCCW}$ & Catalytic & E55 \\
\hline $116 s \_a$ & $\mathrm{CCCW}$ & Catalytic & D117 \\
\hline 2bz1_a & $\mathrm{CCCw}$ & Structural & - \\
\hline 406o_b & $\mathrm{CCCw}$ & Structural & - \\
\hline 2gOd_a & $\mathrm{CCHw}$ & Catalytic & - \\
\hline \multicolumn{4}{|c|}{ Eukaryota } \\
\hline 3v4k_a & $\mathrm{CCHw}$ & Catalytic & E259 \\
\hline 1is8_c & $\mathrm{CCHw}$ & Catalytic & E133 \\
\hline 416o_a & $\mathrm{CCHw}$ & Catalytic & D738 \\
\hline 2w4l_a & $\mathrm{CCHw}$ & Catalytic & E86 \\
\hline 3eyx_a & $\mathrm{CCHw}$ & Catalytic & D59 \\
\hline 5b5z_a & $\mathrm{CCHw}$ & Catalytic & D48 \\
\hline 1zy7_a & $\mathrm{CCHw}$ & Catalytic & E396 \\
\hline 3dh1_a & $\mathrm{CCHw}$ & Catalytic & E73 \\
\hline 5k5w_a & $\mathrm{CCHw}$ & Catalytic & D122 \\
\hline 3e6u_d & $\mathrm{CCHw}$ & Structural & - \\
\hline 2w3q_a & $\mathrm{CCHw}$ & Catalytic & D70 \\
\hline 3vhs_a & $\mathrm{CCHw}$ & Structural & - \\
\hline 3boc_a & $\mathrm{CCHw}$ & Catalytic & D265 \\
\hline 1ekj_c & $\mathrm{CCHw}$ & Catalytic & D162 \\
\hline 1r5t_a & $\mathrm{CCCw}$ & Catalytic & E63 \\
\hline 1h7n_a & $\mathrm{CCCw}$ & Catalytic & D131 \\
\hline 2z3g_a & $\mathrm{CCCw}$ & Catalytic & E56 \\
\hline $\begin{array}{c}\text { 1du3_k } \\
\text { Virus }\end{array}$ & $\mathrm{CCCw}$ & Structural & - \\
\hline 3sv6_a & $\mathrm{CCCW}$ & Structural & - \\
\hline 2g9t_e & $\mathrm{CCCW}$ & Structural & - \\
\hline
\end{tabular}


Table S3. PDB entries of proteins with consensus sequence/structural motifs of $\mathrm{Zn}-\mathrm{CC}(\mathrm{C} / \mathrm{H}) \mathrm{w}$ sites

\begin{tabular}{|c|c|c|}
\hline PDB ID & Zn ligands & $(\underline{\mathrm{H} / \mathrm{C}}) \mathrm{AEx}_{2}(\mathrm{~A} / \mathrm{V}) \mathrm{x}_{35} \mathrm{P} \underline{\mathrm{C}} \times \mathrm{x}(\underline{\mathrm{C} / \mathrm{H}})$ \\
\hline 1r5t_a & $\mathrm{CCCW}$ & C61, E63, C96, C99 \\
\hline 1wkq_a & $\mathrm{CCHw}$ & H53, E55, C83, C86 \\
\hline 1wwr_a & $\mathrm{CCHw}$ & H52, E54, C82, C85 \\
\hline $2 b 3 j \_a$ & $\mathrm{CCHw}$ & $\mathrm{H} 53, \mathrm{E} 55, \mathrm{C} 83, \mathrm{C} 86$ \\
\hline 2g84_a & $\mathrm{CCHw}$ & H77, E79, C112, C115 \\
\hline $2 h x v \_a$ & $\mathrm{CCHw}$ & H49, E51, C77, C86 \\
\hline 2w4l_a & $\mathrm{CCHw}$ & H84, E86, C110, C113, \\
\hline $2 z 3 g \_a$ & $\mathrm{CCCw}$ & C54, E56, C88, C91 \\
\hline 3dh1_a & $\mathrm{CCHw}$ & H71, E73, C107, C110 \\
\hline \multirow[t]{2}{*}{ 3dmo_a } & $\mathrm{CCCW}$ & C53, E55, C88, C91 \\
\hline & & $\underline{\mathrm{C}} \times \mathrm{D} \times \mathrm{R}_{77} \underline{\mathrm{H}} \times x \underline{\mathrm{C}}$ \\
\hline 1ekj_c & $\mathrm{CCHw}$ & C160, D162, H220, C223 \\
\hline $1 g 5 c \_a$ & $\mathrm{CCHw}$ & C32, D34, H87, C90 \\
\hline 2fgy_a & $\mathrm{CCHw}$ & C173, D175, H242, C253 \\
\hline 2w3q_a & $\mathrm{CCHw}$ & C68, D70, H124, C127 \\
\hline 3eyx_a & $\mathrm{CCHW}$ & C57, D59, H112, C115 \\
\hline 3vrk_a & $\mathrm{CCHw}$ & C44, D46, H97, C100 \\
\hline 5swc_a & $\mathrm{CCHw}$ & C39, D41, H98, C101 \\
\hline
\end{tabular}


Table S4. Conserved domains for catalytic and structural $\mathrm{Zn}-\mathrm{CC}(\mathrm{C} / \mathrm{H}) \mathrm{x}$ sites.

\begin{tabular}{|c|c|}
\hline Conserved Domain $^{a}$ & Superfamily Description \\
\hline \multicolumn{2}{|l|}{ Catalytic } \\
\hline A_deamin & Adenosine-deaminase (editase) domain \\
\hline ALAD_PBGS & $\begin{array}{l}\delta \text {-aminolevulinic acid dehydratase (ALAD), also called porphobilinogen } \\
\text { synthase (PBGS) }\end{array}$ \\
\hline APOBEC_N & APOBEC-like N-terminal domain \\
\hline$\beta-\mathrm{CA}$ & $\beta$-Carbonic Anhydrase \\
\hline Cdd & Cytidine deaminase (Nucleotide transport and metabolism) \\
\hline CsoSCA & Carboxysome Shell Carbonic Anhydrase \\
\hline Cytidine_deaminase-like & Cytidine and deoxycytidylate deaminase \\
\hline DHBP_synthase & 3,4-DiHydroxy-2-Butanone 4-Phosphate \\
\hline FrmA & Zn-dependent alcohol dehydrogenase \\
\hline GTP cyclohydrolase II & $\begin{array}{l}\text { GTP cyclohydrolase II catalyzes the first committed step in the biosynthesis of } \\
\text { riboflavin }\end{array}$ \\
\hline LanC like & Lanthionine synthetase C-like protein \\
\hline MafB19-deam & MafB19-like deaminase \\
\hline $\begin{array}{l}\text { Metallohydrolase-like } \\
\text { MBL-fold }\end{array}$ & $\begin{array}{l}\text { Mainly hydrolytic enzymes and related proteins which carry out various } \\
\text { biological functions }\end{array}$ \\
\hline MutL & MutL protein \\
\hline P-loop_NTPase & P-loop containing Nucleoside Triphosphate Hydrolases \\
\hline PRK05222 & 5-methyltetrahydropteroyltriglutamate--homocysteine S-methyltransferase \\
\hline S-methyl trans & Homocysteine S-methyltransferase \\
\hline Tfold & Tunnelling fold \\
\hline tRNA_synt_1e & tRNA-synt 1e domain containing protein \\
\hline TS_Pyrimidine_HMase & Thymidylate synthase and pyrimidine hydroxymethylase \\
\hline UR0-D_CIMS_like & $\begin{array}{l}\text { The URO-D_CIMS like protein superfamily includes bacterial and eukaryotic } \\
\text { uroporphyrinogen decarboxylases (URO-D), coenzyme M methyltransferases } \\
\text { and other putative bacterial methyltransferases, as well as cobalamine (B12) } \\
\text { independent methionine synthases. }\end{array}$ \\
\hline \multicolumn{2}{|r|}{ 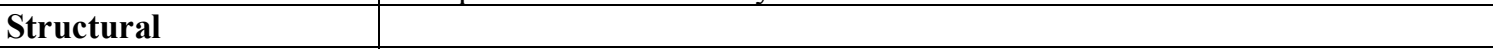 } \\
\hline Arena nucleocapsid & Arenavirus nucleocapsid N-terminal domain \\
\hline AE_Prim_S_like & $\begin{array}{l}\text { Domain similar to that found in the small subunit of } \\
\text { archaeal and eukaryotic DNA primases }\end{array}$ \\
\hline Csc2_I-D & CRISPR/Cas system-associated protein Csc2 \\
\hline Flavi_NS5 & Flavivirus RNA-directed RNA polymerase \\
\hline FmdE & Molybdenum formylmethanofuran dehydrogenase operon \\
\hline HIT like & histidine triad motif-containing proteins \\
\hline IscU_like & Iron-sulfur cluster scaffold-like proteins \\
\hline LIM & LIM domain \\
\hline Maelstrom & piRNA pathway germ-plasm component \\
\hline MOZ_SAS & $\begin{array}{l}\text { This region of these proteins has been suggested to be homologous to } \\
\text { acetyltransferases }\end{array}$ \\
\hline NSP10 & RNA synthesis protein NSP10 \\
\hline OprD & Outer membrane porin, OprD family \\
\hline Peptidase_C19 & Peptidase C19 contains ubiquitinyl hydrolases \\
\hline Peptidase_S29 & Hepatitis C virus NS3 protease \\
\hline PHA00733 & Hypothetical protein \\
\hline PRK12267 & methionyl-tRNA synthetase \\
\hline RecR & RecR protein \\
\hline RING_Ubox & Really Interesting New Gene and U-box domains \\
\hline SET & SET domains are protein lysine methyltransferase enzymes. \\
\hline TNF & Tumor necrosis factor \\
\hline V4R & vinyl 4 reductase domain \\
\hline XylR N & Activator of aromatic catabolism \\
\hline Zf-C2HE & $\mathrm{C} 2 \mathrm{HE} / \mathrm{C} 2 \mathrm{H} 2$ / C2HC zinc-binding finger \\
\hline
\end{tabular}




\begin{tabular}{|l|l|}
\hline ZnF_Rad18 & Rad18-like CCHC zinc finger \\
\hline Catalytic \& Structural & \\
\hline MDR & $\begin{array}{l}\text { Medium chain reductase/dehydrogenases (MDR)/zinc-dependent alcohol } \\
\text { dehydrogenase-like family }\end{array}$ \\
\hline
\end{tabular}

${ }^{a}$ Conserved protein domain family name according to the CDD. ${ }^{32}$ 
Table S5. The mean BVS values of catalytic/structural $\mathrm{Zn}-\mathrm{CC}(\mathrm{C} / \mathrm{H})(\mathrm{D} / \mathrm{E})$ sites.

\begin{tabular}{|c|c|c|c|}
\hline \multicolumn{4}{|c|}{ Structural Zn sites ${ }^{a}$} \\
\hline Set & $\begin{array}{c}\text { \# of } \\
\text { Zn-sites }\end{array}$ & $\begin{array}{c}\text { Mean BVS } \\
\pm \text { standard deviation }\end{array}$ & PDB IDs ${ }^{b}$ \\
\hline 1 & 39 & $1.80 \pm 0.28$ & 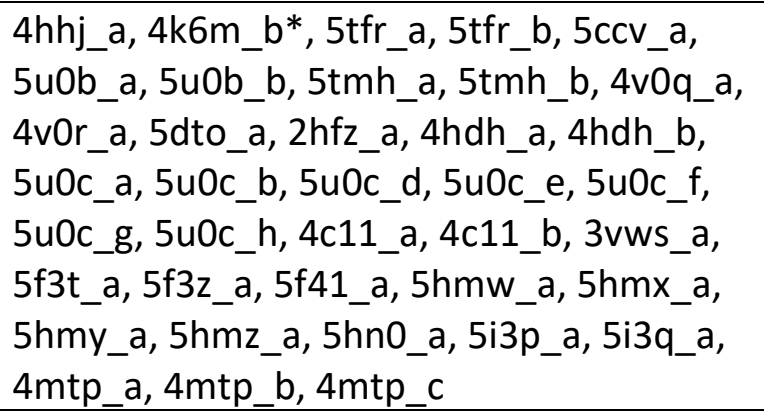 \\
\hline 2 & 26 & $2.08 \pm 0.27$ & 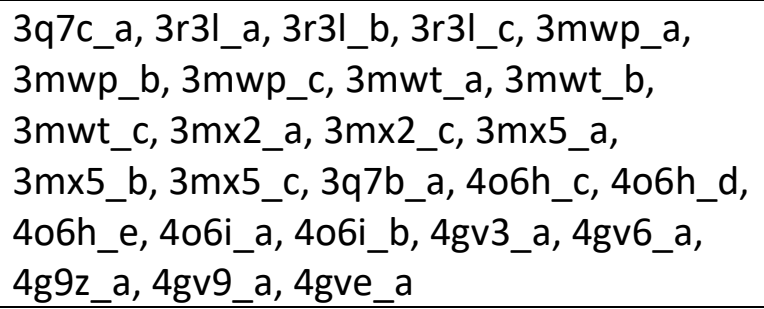 \\
\hline 4 & 19 & $2.18 \pm 0.22$ & $\begin{array}{l}\text { 1rut_x*, 4jcj_c*, 4kfz_a*, 4kfz_b*, 2xjy_a*, } \\
2 x j z \_e^{*}, 21 x d \_a, 214 z \_a, 1 m 3 v \_a, 1 j 2 o \_a, \\
216 y \_b, 216 z \_c, 2 m b v \_a\end{array}$ \\
\hline 3 & 18 & $2.12 \pm 0.21$ & $\begin{array}{l}\text { 5fru_a, 5fru_b, 5frv_a, 5frv_b, 5frw_a, } \\
5 f r w \_b, 5 f r x \_a, 5 f r y \_a, 5 f r y \_b, 5 f r z \_a, \\
5 f r z \_b, 5 f s 0 \_a, 5 f s 0 \_b, 5 k b e \_b, 5 k b g \_b, \\
5 k b h \_a, 5 k b h \_b, 5 k b i \_a\end{array}$ \\
\hline 5 & 13 & $1.88 \pm 0.14$ & $\begin{array}{l}\text { 4xba_a, 3szq_a, 3sp4_a, 3sp4_b, 3spd_a, } \\
\text { 3spd_b, 3spd_c, 3spd_d, 3spl_a, 3spl_b, } \\
\text { 3spl_c, 3spl_d, 4ykl_b }\end{array}$ \\
\hline 6 & 10 & $1.70 \pm 0.42$ & $\begin{array}{l}\text { 2rgt_a*,2jtn_a*, 2rgt_b*, 3mmk_a*, } \\
3 m m k \_b^{*}\end{array}$ \\
\hline 7 & 7 & $1.96 \pm 0.33$ & $\begin{array}{l}\text { 1u6g_b, 3rtr_b, 3rtr_d, 3rtr_f, 3rtr_h, } \\
2 \operatorname{lgv} a, 1 / d j \_b\end{array}$ \\
\hline 8 & 4 & $1.98 \pm 0.07$ & 4hi8_b, 3f6q_b, 3ixe_b, 4hi9_b \\
\hline 9 & 3 & $1.74 \pm 0.03$ & 5afo_c, 5afo_b, 5afo_d \\
\hline 10 & 3 & $2.06 \pm 0.2$ & 2dar_a, 2dlo_a, 2dj7_a \\
\hline 11 & 2 & $2.60 \pm 0.3$ & 1zt2_a, 1zt2_c \\
\hline 12 & 2 & $2.04 \pm 0.5$ & 2wbt_a, 2wbt_b \\
\hline 13 & 1 & 2.13 & 4ybg_a \\
\hline 14 & 15 & $2.09 \pm 0.04$ & 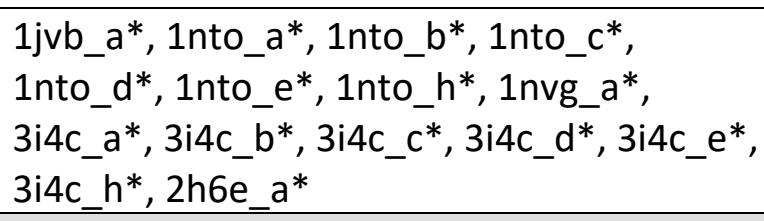 \\
\hline \multicolumn{4}{|c|}{ Catalytic Zn sites ${ }^{c}$} \\
\hline Set & $\begin{array}{c}\text { \# of } \\
\text { Zn-sites }\end{array}$ & $\begin{array}{c}\text { Mean BVS } \\
\pm \text { standard deviation }\end{array}$ & PDB IDs ${ }^{b}$ \\
\hline
\end{tabular}




\begin{tabular}{|c|c|c|c|}
\hline 1 & 64 & $2.07 \pm 0.29$ & $\begin{array}{l}\text { 5cxk_a, 2a8c_a, 2a8c_b, 2a8c_c, 2a8c_d, } \\
\text { 2a8c_e, 2a8c_f, 2a8d_a, 2a8d_b, 2a8d_c, } \\
\text { 2a8d_d, 2a8d_e, 2a8d_f, 3e24_a, 3e28_b, } \\
\text { 3e28_c, 3e28_d, 3e2a_a, 3e2a_b, 3e2a_c, } \\
\text { 3e2a_d, 3e2a_e, 3e2a_f, 3e2w_a, 3e2w_b, } \\
\text { 3e2w_c, 3e2w_d, 3e3f_b, 3e3g_a, 3e3g_c, } \\
\text { 3e3g_d, 3e3g_e, 3e3g_f, 3e3i_a, 3e3i_b, } \\
\text { 3e3i_c, 3e3i_d, 3e3i_e, 3e3i_f, 3e3i_g, } \\
\text { 3e3i_h, 3e3i_i, 3e3i_j, 3e3i_k, 3e3i_l, } \\
\text { 4wak_a, 4wak_b, 4wam_b, 5cxk_b, 5cxk_c, } \\
\text { 5cxk_d, 5cxk_e, 5cxk_f, 5cxk_g, 5cxk_h, } \\
\text { 1i6o_a, 1i6o_b, 1i6p_a, 1t75_a, 1t75_b, } \\
\text { 1t75_d, 1t75_e, 2esf_a, 2esf_b }\end{array}$ \\
\hline 2 & 34 & $1.81 \pm 0.12$ & $\begin{array}{l}\text { 1h2b_b, 1jvb_a*, 1nto_a*, 1nto_b*, } \\
\text { 1nto_c*, 1nto_d*, 1nto_e } \text { e }^{*}, 1 \text { nto_h*, } \\
\text { 1nvg_a*, 1r37_a, 1r37_b, 2eer_a, 2eer_b, } \\
\text { 3i4c_a*, 3i4c_b*, 3i4c_c*, 3i4c_d*, 3i4c_e*, } \\
\text { 3i4c_h*, 2h6e_a* }\end{array}$ \\
\hline 3 & 19 & $1.56 \pm 0.34$ & $\begin{array}{l}\text { 3s2e_a, 4eex_b, 4eez_b, 3s1l_a, 3s1l_b, } \\
3 s 1 l \_c, 3 s 1 l \_d, 3 s 2 e \_b, 3 s 2 e \_c, 3 s 2 e \_d, \\
3 s 2 f \_a, 3 s 2 f \_b, 3 s 2 f \_c, 3 s 2 f \_d, 3 s 2 g \_a, \\
3 s 2 g \_b, 3 s 2 g \_c, 3 s 2 i \_b, 3 s 2 i \_d\end{array}$ \\
\hline 4 & 12 & $1.74 \pm 0.35$ & $\begin{array}{l}\text { 4dl9_a, 4dl9_b, 4gl4_a, 4gl4_b, 4jji_b, } \\
\text { 4l0q_a, 4l0q_b, 3uko_a, 3uko_b, 1ma0_a, } \\
\text { 1ma0_b, 1mp0_a, }\end{array}$ \\
\hline 5 & 10 & $2.10 \pm 0.07$ & $\begin{array}{l}5 f i 3 \_a, 5 f i 3 \_b, 5 f i 5 \_a, 5 f i 5 \_b, 5 h 81 \_a, \\
5 h 81 \_b, 5 h 82 \_a, 5 h 82 \_b, 5 h 83 \_a, 5 h 83 \_b\end{array}$ \\
\hline 6 & 5 & $2.06 \pm 0.13$ & 3wid_a, 3wid_b, 3wid_c, 3wid_d, 3wie_b \\
\hline 7 & 4 & $2.21 \pm 0.20$ & $1 d d z_{-} a^{*}, 1 d d z_{-} b^{*}$ \\
\hline 8 & 2 & $1.70 \pm 0.01$ & 3bq6_a, 3bq6_b \\
\hline 9 & 2 & $2.10 \pm 0.67$ & 1li5_a, 1li5_b \\
\hline 10 & 2 & $1.82 \pm 0.08$ & 2nyt_a, 2nyt_c \\
\hline 11 & 2 & $1.50 \pm 0.64$ & 3ppg_a, 4ztx_a \\
\hline 12 & 1 & 1.95 & $3 w r g \_a$ \\
\hline 13 & 1 & 1.85 & 1ym3_a \\
\hline 14 & 1 & 1.16 & $4 w 6 z \_b$ \\
\hline 15 & 1 & 1.16 & 4ay7_b \\
\hline 16 & 1 & 1.89 & 1vq2_a \\
\hline
\end{tabular}

astructural Zn-sites whose mean BVS minus standard deviation is $<2$ are highlighted in italics. "PDB entries with $2 \mathrm{Zn}$ sites are labeled "*".

cCatalytic Zn-sites whose mean BVS minus standard deviation is $>2$ are highlighted in italics. 
Electronic energies of all the stationary points

Energies of the system with 2nd-shell Asp $(M 11 / 6-31+G(3 d, p))$ :

CCCw_asp $10 \mathrm{E}(\mathrm{RM} 11)=-3474.72450375$

CCCh_asp $10 \mathrm{E}(\mathrm{RM} 11)=-3474.70976436$

CCCW_asp $30 E(R M 11)=-3474.74371904$

CCCh_asp $30 \mathrm{E}(\mathrm{RM} 11)=-3474.73166128$

CCHw_asp $10 \mathrm{E}(\mathrm{RM} 11)=-3262.69244708$

CCHh_asp $10 \mathrm{E}(\mathrm{RM} 11)=-3262.69307205$

CCHw_asp $30 \mathrm{E}(\mathrm{RM} 11)=-3262.70460028$

CCHh_asp $30 E(R M 11)=-3262.70409909$

Energies of the system without 2nd-shell Asp $(M 11 / 6-31+G(3 d, p))$ :

CCCW $10 \mathrm{E}(\mathrm{RM} 11)=-3246.16122955$

CCCh $10 E(R M 11)=-3245.67448959$

CCCW $30 E(R M 11)=-3246.16847326$

CCCh $30 E(R M 11)=-3245.69502804$

$\mathrm{CCHw} 10 \mathrm{E}(\mathrm{RM} 11)=-3034.12420361$

$\mathrm{CCHh} 10 \mathrm{E}(\mathrm{RM} 11)=-3033.65097894$

$\mathrm{CCHw} 30 \mathrm{E}(\mathrm{RM} 11)=-3034.12887833$

$\mathrm{CCHh} 30 \mathrm{E}(\mathrm{RM} 11)=-3033.66370854$

SI information II. The Cartesian coordinates of all the structures used in the figure, fully optimized using M06-2X/SDD at epsilon 10 and 30.

CCCh_asp_epsi10.xyz

e/opt.log Energy: -1922.87050938

Zn1 $110.171363-21.75344390 .345171$

C2 $108.650870-20.11446092 .724686$

C3 $106.206210-17.35516089 .791384$

C4 $107.050647-18.59315789 .633895$

O5 $108.216157-18.58312389 .187200$

$06106.419734-19.71311490 .031074$

C7 $111.964257-23.20049487 .988251$

S8 $111.794344-23.47704089 .847540$

C9 107.900904-22.59689188.093902

S10 107.801271-22.396941 89.961426

$011110.509018-20.36521589 .020809$

$012113.023138-20.32876189 .298861$

H13 107.809635 - 20.78652192 .534258

H14 106.721099 -16.493382 89.368365

H15 $106.013980-17.18360190 .854565$

H16 105.242053 -17.498977 89.297912

H17 $113.021326-23.23309687 .711247$

H18 $106.960379-23.00532087 .717654$

H19 108.716665 -23.281340 87.847922 
H20 108.097167 -21.629691 87.626049

H21 111.552686 -22.219952 87.737570

H22 $113.151421-21.23285289 .651943$

H23 $112.011056-20.21674589 .134985$

H24 111.425924 -23.981810 87.445643

H25 109.807306 - 19.68467088 .988250

S26 110.277127-21.061814 92.646976

H27 108.540033 -19.670864 93.717671

H28 108.644159 -19.317402 91.974585

H29 106.961070 -20.604164 90.002206

CCCh_asp_epsi30.xyz

e/opt.log Energy: -1922.87081436

Zn1 110.110123 -21.723847 90.234822

C2 108.651685-20.003049 92.676164

C3 106.255599-17.377087 89.936109

C4 $107.039520-18.63116089 .654627$

O5 $108.166430-18.63994889 .116752$

O6 $106.404757-19.74985890 .052864$

C7 $112.085948-23.26854188 .050788$

S8 $111.777765-23.44324389 .903864$

C9 $107.831728-22.62085988 .007886$

S10 107.773209-22.451406 89.881150

$011110.472464-20.36595188 .882522$

$012112.954974-20.28186889 .395109$

H13 107.795189-20.631815 92.419012

H14 106.753262 -16.515287 89.493330

H15 106.177324 -17.239203 91.018935

H16 105.242683 -17.473815 89.538698

H17 113.157152 -23.352501 87.852277

H18 106.899086 -23.062979 87.651923

H19 $108.668404-23.26506687 .726497$

H20 $107.971920-21.63838287 .552106$

H21 111.730063 -22.290639 87.719299

H22 113.072120 -21.189006 89.745082

H23 111.964725 -20.189403 89.130135

H24 111.557981 -24.056447 87.508730

H25 109.756984 - 19.70029488 .832012

S26 110.253031-20.982024 92.524014

H27 108.543487 -19.651363 93.705070

H28 108.670131 - 19.13924392 .005126

H29 106.923254 -20.644715 89.968097

CCCh_epsi10.xyz

opt.log Energy: -1693.88191892

Zn1 $0.564297-0.597159-1.563574$

O2 -1.208992 -1.337310 -1.964359 
C3 $2.2680722 .076740-2.486550$

H4 2.518836 2.938759-3.110292

H5 -1.474181 -2.117464 -1.443939

S6 $0.7273141 .237784-3.162783$

H7 $3.1065951 .374736-2.486388$

H8 $2.0883232 .408391-1.459234$

S9 0.7224550 .3049650 .679610

C10 -0.081264 1.995342 0.471016

H11 -1.126655 1.9585700 .785830

H12 0.4546882 .7285701 .080191

H13 -0.039873 2.292592 -0.581678

S14 2.339154 -2.229814 -1.776723

C15 $1.930635-2.979853-3.455489$

H16 1.756260 -2.188724 -4.189939

H17 $2.769326-3.597509-3.787213$

H18 $1.034548-3.601482-3.386709$

H19 -2.041678 -0.016874 -1.967038

O20 -2.400895 $0.938796-2.010399$

H21 -1.636967 $1.517943-2.209341$

\section{CCCh_epsi30}

opt.log Energy: - 1693.88212180

Zn1 $0.499583-0.607538-1.572745$

O2 -1.264930 -1.346953 -2.019044

C3 $2.2277962 .068365-2.483600$

H4 2.474603 2.932247 -3.105593

H5 -1.528259 -2.167702 -1.565105

S6 $0.6887001 .227425-3.161636$

H7 $3.0703411 .371484-2.486730$

H8 2.047606 2.399524 -1.456636

S9 0.6469120 .2741910 .683691

C10 -0.0114282 .0281350 .495333$

H11 -1.013172 2.103487 0.924988

H12 0.654244 2.723871 1.013180

H13 -0.057931 2.290354 -0.566234

S14 $2.276102-2.249283-1.728445$

C15 $1.982587-2.977214-3.440854$

H16 $1.861251-2.177605-4.176513$

H17 2.841687 -3.591379 -3.721496

H18 1.084755 -3.599681 -3.442524

H19 - 2.092259 -0.030470 -1.988654

O20 -2.441532 $0.929661-2.011436$

H21 -1.676654 $1.506080-2.214947$

CCCw_asp_epsi10

a/opt.log Energy: -1922.88566364 
Zn1 $110.547918-21.98040689 .896842$ C2 106.776997-18.116832 87.403685 C3 $107.999268-19.00927987 .298784$ O4 $108.750893-19.05849888 .379497$ O5 $108.251990-19.66270186 .242244$ C6 $112.958777-23.04089087 .984226$ S7 $112.749969-22.95222089 .855454$ C8 $107.537888-22.38981288 .569250$ S9 $108.838651-23.54809489 .288560$ $010110.556840-20.66428488 .294308$ $011113.037216-19.77802189 .072742$ H12 $107.071693-17.12522787 .756830$ H13 106.087591 - 18.54044188 .141379 H14 106.273512 -18.037109 86.439401 H15 $113.891512-23.55966287 .751611$ H16 107.167564 -21.707902 89.339866 H17 106.705685 -22.987723 88.188592 H18 107.961452 -21.800508 87.751811 H19 112.997120 -22.028487 87.571686 H20 113.289134 -20.584930 89.573211 H21 112.129554 -19.939952 88.710753 H22 112.125638 - 23.58706787 .533506 S23 110.134846-20.704149 91.857491 C24 108.252957-20.750547 91.917126 H25 107.923271 -20.494518 92.927063 H26 107.837966 -20.030472 91.207365 H27 107.898799 -21.753405 91.661002 H28 109.720148 -19.893530 88.343178 H29 110.551155 -21.103334 87.422537

CCCw_asp_epsi30

a/opt.log Energy: -1922.88546300 Zn1 110.551921 -21.970106 89.854509 C2 $106.837412-18.07988287 .274902$ C3 108.025819-19.018328 87.209445 O4 $108.797096-19.02842488 .280471$ O5 $108.238308-19.74924086 .196382$ C6 $112.978804-23.06896687 .975942$ S7 $112.750092-22.94704989 .843226$ C8 107.409005 -22.387496 88.819251 S9 $108.824223-23.53512489 .305076$ $010110.557705-20.68533088 .233420$ $011113.030554-19.78140389 .006548$ H12 107.159358 -17.093069 87.616065 H13 $106.117790-18.46612588 .004475$ H14 106.354313 -18.001296 86.300244 H15 113.922348 -23.575652 87.762581 
H16 106.592126 -22.485580 89.538558 H17 107.046519 -22.655537 87.824001 H18 107.749916 -21.348350 88.805713 H19 $113.003210-22.06482387 .543157$ H20 113.292083 -20.582172 89.511886 H21 112.132405 -19.963192 88.629472 H22 $112.159391-23.63780487 .529119$ S23 110.161278-20.634500 91.781964 C24 108.287832 -20.74033791.946622 H25 108.017530 -20.666224 93.002605 H26 107.811806 -19.926184 91.395074 H27 107.938452 -21.696419 91.547304 H28 109.739812 -19.891806 88.263811 H29 110.538893 -21.146582 87.373177

\section{CCCw_epsi10}

opt.log Energy: -1694.35853259

Zn1 $0.559041-1.010730-0.597740$

O2 -1.213139-0.188228 -1.272891

C3 $1.6689902 .009489-0.919396$ H4 $0.7530292 .271702-1.454930$ H5 -1.554491 $0.601046-0.729797$ S6 2.303172 $0.333580-1.499050$ H7 $2.4326582 .765078-1.118572$ H8 1.4583151 .9827030 .154393 S9 $0.247414-0.4393581 .756624$ C10 1.9403330.211467 2.251364 H11 2.5613530 .3296451 .358966 H12 1.8283261 .1748252 .753864 H13 2.414369 -0.497651 2.932597 H14 -1.872507 -0.683891 -1.783641 S15 $0.491229-3.269467-1.304331$ C16 1.770677 -3.225194-2.686835 H17 2.721717 -2.842754 -2.309116 H18 1.914582 -4.241204 -3.061444 H19 $1.429840-2.585398-3.503605$ O20 -1.658886 1.6114960 .420641 H21 - 1.6138982 .5815280 .386574 H22 -1.018126 1.1993161.070325

\section{CCCw_epsi30}

opt.log Energy: -1694.35866094 Zn1 $0.553314-1.012383-0.572879$ O2 -1.230943 -0.241028 -1.268415 C3 $1.6964242 .025405-0.898932$ H4 0.7920852 .327055 -1.433056 H5 -1.555861 $0.570680-0.744851$ 
S6 $2.2789080 .340233-1.506300$

H7 2.484866 2.759157 -1.081410

H8 1.4799221 .9858460 .172937

S9 $0.246753-0.4279701 .775944$

C10 1.9454970.209983 2.269332

H11 2.5699100 .3152711 .377837

H12 1.8406391 .1791402 .761568

H13 2.411494 -0.496460 2.958785

H14 - $1.907246-0.741345-1.752336$

S15 $0.622565-3.303076-1.189206$

C16 1.733428 -3.215034-2.709701

H17 $2.653589-2.675286-2.474498$

H18 $1.982738-4.232529-3.018895$

H19 1.220904 -2.706118 -3.528665

$020-1.6418731 .6117820 .369537$

H21 -1.610094 2.5806730 .299628

H22 -1.003019 1.234005 1.037577

\section{CCHh_asp_epsi10}

e/opt.log Energy: -1710.83817097 Zn1 $109.878872-21.67597690 .159424$

C2 $108.827482-20.37319892 .730763$

C3 109.112697 -19.374760 93.636061

N4 109.879335 -20.46448891.815881

C5 $110.782227-19.53569992 .152694$

N6 110.348319-18.858479 93.253948

C7 $106.520097-17.46064689 .201510$

C8 107.540177-18.43104988.648805

O9 $108.670800-18.49386789 .344046$

$010107.326020-19.11918887 .615429$

C11 112.135198 -23.163944 88.309641

S12 $111.838907-23.06147390 .167782$

C13 107.554403 -22.563513 88.032713

S14 107.681944-22.512079 89.914913

$015110.216018-20.24663288 .758155$

$016112.704042-19.92719689 .772234$

H17 107.962292 -21.011279 92.663609

H18 108.563092 -19.002519 94.482114

H19 111.701502 -19.344381 91.617259

H20 110.841294 -18.100848 93.708498

H21 106.985201 -16.483925 89.356999

H22 106.171083 -17.822887 90.173251

H23 105.673053 -17.368841 88.521507

H24 113.072057 -23.695751 88.131827

H25 106.605731 - 23.03478287 .765481

H26 108.373052 -23.153546 87.612311

H27 107.579871 -21.547155 87.632466 
H28 $110.139313-20.52002787 .825584$

H29 112.211245 -22.158583 87.885873

H30 112.833217-20.853692 90.077251

H31 111.833563 -19.902386 89.288454

H32 111.319946 -23.703571 87.822726

H33 109.426952 -19.322639 89.025790

CCHh_asp_epsi30

e/opt.log Energy: -1710.83824746

Zn1 109.851513-21.64822290.146996

C2 108.852997-20.394927 92.760862

C3 109.156461 -19.41451193.680100

N4 109.880565 -20.45826591.815436

C5 $110.786702-19.53063192 .150157$

N6 110.377427 -18.881724 93.276734

C7 $106.540750-17.35597989 .201923$

C8 $107.510268-18.37419188 .643568$

O9 $108.657972-18.45844889 .307413$

$010107.244001-19.07808887 .632668$

C11 112.116191-23.130241 88.272941

S12 $111.794368-23.06372690 .129049$

C13 107.564575 -22.713118 88.068889

S14 107.640412 -22.466957 89.937404

$015110.186151-20.22741388 .736723$

$016112.683729-19.92491789 .737249$

H17 107.992041 -21.040215 92.708072

H18 108.628697 -19.065780 94.549756

H19 111.692905 -19.323086 91.599349

H20 110.878830 -18.13300193.737724

H21 107.026663 -16.378164 89.258961

H22 106.258643 -17.645724 90.218554

H23 105.648774 - 17.29236388 .578580

H24 113.051264-23.665859 88.097818

H25 106.619801-23.201425 87.820723

H26 108.390260 -23.345868 87.734364

H27 107.613276 - 21.74450387 .566442

H28 110.133445 -20.513815 87.806292

H29 112.206087 -22.117323 87.870930

H30 112.808571 -20.849079 90.049885

H31 111.808618 -19.895060 89.262674

H32 $111.303918-23.65255987 .763474$

H33 109.404122 -19.304819 88.985290

CCHh_epsi10

opt.log Energy: -1481.83779491

Zn1 -0.039481-0.494911 -0.884885

O2 -0.070530 -2.419728 -1.134212 
C3 $2.0100471 .546223-2.492918$

H4 $2.1437722 .544654-2.915939$

H5 -0.311688 -2.819656 -1.986181

S6 $0.2121361 .028776-2.698287$

H7 $2.6681010 .845830-3.012172$

H8 2.270752 $1.559172-1.431075$

C9 -2.639009-0.759188 0.717111

C10 -2.701534 $1.104381-0.456294$

H11 -2.299201 -1.693907 1.143913

C12 -3.871278 1.0583640 .273085

H13 -2.345992 $1.849639-1.148448$

H14 -4.702673 1.7383660 .329964

N15 -1.946771 -0.035603 -0.170246

N16 -3.813596 -0.124610 1.006810

H17 -4.518903 -0.459158 1.650333

S18 $1.381415-0.0523381 .013330$

C19 0.712468 1.657261 1.426339

H2O -0.366075 1.6088571 .602383

H21 1.2074662 .0311782 .325652

H22 0.9052892 .3454910 .598158

H23 - $0.374234-2.8554090 .317981$

O24 -0.508851-2.871632 1.332285

H25 0.011369 -2.126054 1.701314

\section{CCHh_epsi30}

opt.log Energy: -1481.83594706

Zn1 -0.129615 -0.384876 -0.915605

O2 -0.100845 -2.250191-1.453178

C3 $2.1370131 .572946-2.292866$

H4 $2.4257852 .540493-2.709323$

H5 -0.438434 - 2.520014-2.324542

S6 $0.2778021 .350438-2.498747$

H7 $2.6761410 .781282-2.817601$

H8 2.399453 1.540464 -1.231968

C9 -2.386430 -0.110832 1.118971

C10 -3.143051 $0.535163-0.841398$

H11 - $1.743108-0.4669961 .906360$

C12 -4.183747 0.6828260 .051661

H13 -3.115513 $0.748542-1.896431$

H14 -5.192037 $1.032959-0.080107$

N15 -2.029817 $0.037739-0.160850$

N16 -3.685818 0.270565 1.284068

H17 -4.197224 0.2562932 .157446

S18 $1.194161-0.2464151 .099946$

C19 0.8605351 .5521291 .538401

H2O -0.210813 1.712131 1.692582

H21 1.3969441 .8060342 .455234 
H22 1.2012792 .2029390 .728584

H23 - $0.382180-2.910853-0.065412$

O24 -0.489455 -3.137145 0.922428

H25 -0.128021 -2.389622 1.440347

\section{CCHw_asp_epsi10}

a/opt.log Energy: -1710.83424484

Zn1 $109.519693-21.94371889 .689835$

C2 109.534354 -19.813440 91.980207

C3 $110.118712-19.57935793 .209681$

N4 109.952407 -21.070947 91.526661

C5 $110.762989-21.58366592 .455295$

N6 110.886941 -20.70667393.493959

C7 $105.885718-18.77147488 .466768$

C8 $107.385313-18.76591788 .708763$

O9 $107.848341-18.37514789 .831475$

$010108.128143-19.20787887 .726714$

C11 $112.672902-22.64705789 .474254$

S12 111.117704 -23.703500 89.602131

C13 106.879245 -22.323396 87.721580

S14 107.195404 -22.312828 89.578274

$015110.123960-20.42713388 .520691$

$016111.705458-18.82121889 .952470$

H17 108.861333 -19.204097 91.387727

H18 110.046808 -18.743766 93.883632

H19 111.246263 -22.54546192.391693

H20 111.443294 -20.853056 94.326366

H21 105.372526 - 18.11172089 .168807

H22 105.535049 -19.799400 88.623013

H23 105.661756 - 18.48499387 .436852

H24 $113.545882-23.30288489 .439129$

H25 $105.810233-22.47582987 .552857$

H26 107.434214 -23.134836 87.244821

H27 107.185856 -21.363538 87.299659

H28 109.313379 - 19.86255888 .135288

H29 112.758615 -21.984809 90.341381

H30 $111.243930-18.69427190 .803908$

H31 112.189164 -18.021950 89.679944

H32 110.812003 -19.850769 88.946517

H33 112.640411 -22.037716 88.568647

CCHw_asp_epsi30

a/opt.log Energy: -1710.83517465

Zn1 $109.502981-21.90727989 .716875$

C2 109.575354 -19.767040 91.952372 
C3 $110.173869-19.50407093 .169038$ N4 $109.947800-21.05536591 .548345$ C5 $110.747521-21.55685192 .493453$ N6 110.905808-20.64393993.494402 C7 $105.739636-18.78995988 .433891$ C8 107.225811 -18.772680 88.749409 O9 $107.632105-18.41966489 .905849$ $010108.021541-19.15599187 .783850$ C11 112.675679-22.664284 89.469938 S12 $111.083547-23.67143489 .504978$ C13 106.949915 -22.341989 87.686037 S14 107.194178 -22.366567 89.554309 $015110.038766-20.33747388 .590265$ $016111.762462-18.83317589 .921676$ H17 108.915856 -19.159538 91.342922 H18 110.134820 -18.640616 93.809700 H19 111.204411 -22.532583 92.472322 H20 111.462962 -20.774966 94.329311 H21 105.168500 -18.276250 89.208883 H22 105.417965 -19.837269 88.382982 H23 $105.553563-18.33189287 .459148$ H24 113.525253 - 23.34758889 .405107 H25 $105.908228-22.58919287 .466948$ H26 107.599182 -23.079581 87.207940 H27 107.180147 -21.343601 87.307427 H28 109.220882 - 19.78276488 .213712 H29 $112.768969-22.06516690 .380580$ H30 111.482850 -18.708504 90.848189 H31 $112.662223-18.49927089 .762220$ H32 110.750809 -19.770706 88.993221 H33 112.682407 -21.997755 88.604699

\section{CCHw_epsi10}

opt.log Energy: -1482.30845883

Zn1 -0.360071 -0.004930 -0.120632

O2 -0.268821 -1.719726-1.226009

H3 -0.469187 -1.560488 -2.213648

C4 $2.0149931 .193266-2.081730$ H5 2.612224 $1.450994-1.204841$ H6 $0.182370-2.547742-0.999665$ S7 $0.2095031 .637797-1.779423$ H8 2.376641 1.755707 -2.944281 H9 2.108564 $0.122888-2.281479$ C10 -3.149176 0.4923471 .040253 C11 -3.260558 -0.039065 -1.095013 H12 -2.787109 0.706672 2.031021 C13 -4.551420 $0.199080-0.676856$ 
H14 -2.889853 -0.325169 -2.066774 H15 -5.488322 $0.162529-1.203567$

N16 -2.397742 $0.148041-0.010129$ N17 -4.460812 0.5325530 .671938 H18 -5.235960 0.767223 1.279035 S19 $0.752280-0.0895431 .928278$ C20 1.936641 1.3667461.758890 H21 2.0683191 .8231812 .741731 H22 2.9035321 .0197151 .389465 H23 1.5281122 .1069371 .066364 O24 -0.815293 -0.756489-3.455131 H25 - $0.894829-0.946300-4.404103$ H26 - $0.5590250 .181776-3.228693$

\section{CCHw_epsi30}

opt.log Energy: -1482.30849458

Zn1 -0.346121 -0.012898 -0.108824

O2 -0.246141 -1.707298 -1.238215

H3 -0.463643 -1.551047 -2.223136

C4 $2.0143911 .217470-2.085119$ H5 2.613648 $1.469613-1.208001$ H6 -0.125392 -2.626955 -0.955593 S7 $0.2057081 .638504-1.767900$ H8 $2.3656821 .794725-2.942143$ H9 2.116976 $0.151085-2.299731$ C10 -3.147884 0.4836941 .047650 C11 -3.240403 -0.046472 -1.089118 H12 -2.796484 0.6989712 .041956 C13 -4.535464 $0.185566-0.680095$ H14 -2.862523 - $0.330443-2.058953$ H15 -5.468610 $0.145002-1.213166$ N16 -2.385984 0.143726 0.002723 N17 -4.456262 0.5182400 .669233 H18 -5.237425 0.7481051 .270877 S19 0.772174 -0.077549 1.941950 C20 1.955501 1.3776561.756221 H21 2.0923961 .8421722 .734550 H22 2.9206261.028868 1.383932 H23 1.5428322 .1120041 .059957 O24 -0.810831 -0.753398 -3.467642 H25 - $0.814186-0.955099-4.417938$ H26 -0.557580 0.183759 -3.238476 